\title{
Metabolite Profiling of Serum from Patients with Tuberculosis
}

\author{
Hee-Bin Park ${ }^{1 \dagger}$, Min-Gyu Yoo ${ }^{2 \dagger}$, Sangho Choi ${ }^{1}$, Seong-Han Kim ${ }^{1}$, and Hyuk Chu ${ }^{1 *}$ \\ ${ }^{1}$ Division of Bacterial Disease Research, Center for Infectious Diseases Research, National Institute of Infectious Disease (NIID), ${ }^{2}$ Division of \\ Endocrine and Kidney Disease Research, Department of Chronic Disease Convergence Research, Korea National Institute of Health, Korea \\ Disease Control and Prevention Agency, Cheongju 28160, Republic of Korea
}

Received: January 20, 2021 / Revised: March 2, 2021 / Accepted: March 3, 2021

Tuberculosis (TB) is a major infectious disease that threatens the life and health of people globally. Here, we performed a metabolomic analysis of serum samples from patients with intractable TB to identify biomarkers that might shorten the TB treatment period. Serum samples collected at the commencement of patients' treatment and healthy controls were analyzed using the capillary electrophoresis and time-offlight mass spectrometry metabolome analysis method. The analysis identified the metabolites cystine, kynurenine, glyceric acid, and cystathionine, which might be useful markers for monitoring the TB treatment course. Furthermore, our research may provide experimental data to develop potential biomarkers in the TB treatment course.

Keywords: Biomarker, CE-TOFMS, Mycobacterium tuberculosis, metabolites

Tuberculosis (TB) is caused by Mycobacterium tuberculosis (MTB) and is a major health problem globally [1]. MTB has infected 10 million people and caused 1.8 million deaths per year worldwide [2]. However, a rapid and accurate TB diagnosis remains a major challenge for global control efforts [3]. Recently, the incidence of pulmonary tuberculosis is high in immunocompromised individuals, and the emergence of multidrug-resistant bacteria has made diagnosis and treatment more complicated $[4,5]$.

A standard diagnosis method for TB is a smear and culture for acid-fast bacilli in clinical specimens. Although these techniques are important steps for TB diagnosis, they have several drawbacks [6-8]. Smear microscopy is only effective for TB diagnosis when the bacterial load is $>10,000$ organisms/ml of sample [9].

\footnotetext{
*Corresponding author

Tel: +82-43-719-8465, Fax: +82-43-719-8489

E-mail: chuhyuk@korea.kr

${ }^{\dagger}$ These authors contributed equally to this work.
}

Moreover, the positivity rate of a TB sputum smear is only $20-30 \%$. Sputum cultures have been widely used in the diagnosis of MTB, but it can take 4-8 weeks to obtain results. This can lead to delayed treatment in patients with potential TB [10,11]. Therefore, it is necessary to develop biomarkers that can be used for an accurate diagnosis that might lead to early treatment.

Moreover, metabolomics has recently emerged as a potential tool to make striking advances in new biomarker research by enabling multiple profiling and comparing multiple metabolites in a biological sample [12]. Therefore, metabolomics has been widely used to understand biological processes and metabolic behavior pathways of various diseases and to figure out host responses, allowing the researcher to develop advanced diagnostic tools and treatment strategies $[13,14]$.

In this study, we used capillary electrophoresis and time-of-flight mass spectrometry (CE-TOFMS) to profile and compare metabolites found in the serum of patients with active TB versus healthy controls.

Study participants were recruited from an intractable 
TB cohort from 2013 to 2014. Peripheral blood samples were obtained by venipuncture from 7 patients with active TB disease and 7 healthy controls. Blood was collected in serum separating tubes and centrifuged at $1500 \mathrm{rpm}$ for $15 \mathrm{~min}$ at $4^{\circ} \mathrm{C}$ to obtain serum. The serum was immediately frozen and stored at $-80^{\circ} \mathrm{C}$ until analysis. The Institutional Review Board (IRB) of the Korea Disease Control and Prevention Agency (KDCA) approved this study (2019-04-05-2C-A).

Metabolite extraction from samples was carried out according to the protocol provided by Human Metabolome Technologies (HMT, Japan). Briefly, $50 \mu \mathrm{l}$ of thawed serum was treated with methanol $(450 \mu \mathrm{l})$ containing internal standards. Chloroform (450 $\mu \mathrm{l})$ and ultrapure water $(200 \mu \mathrm{l})$ were sequentially added to the serummethanol sample, and the mixture was centrifuged at $2,300 \times g$ at $4^{\circ} \mathrm{C}$ for $5 \mathrm{~min}$. The upper aqueous phase $(800 \mu \mathrm{l})$ was filtered by centrifugation, and the filtrate was concentrated and resuspended in ultrapure water $(25 \mu \mathrm{l})$ for analysis. Cationic compounds and anionic compounds were measured in the CE-TOFMS system (Fused silica capillary, i.d. $50 \mu \mathrm{M} \times 80 \mathrm{~cm}$, Agilent Technologies, Santa Clara, California). Peaks detected by CE-TOFMS were extracted using automatic integration software (MasterHands ver. 2.17.1.11 developed at Keio University) to provide the requisite information, including $\mathrm{m} / z$, migration time (MT), and peak area. Putative metabolites were then assigned from the standard library at HMT and the Known-Unknown peak library based on $m / z$ and MT. The tolerance range for the peak annotation was configured at $\pm 0.5 \mathrm{~min}$ for MT and $\pm 10 \mathrm{ppm}$ for $\mathrm{m} / z$. In addition, all of the target metabolite concentrations were quantified by normalizing the peak area of each metabolite to the area of the internal standard and by using standard curves, which were obtained by single-point $(100 \mu \mathrm{M}$ or $50 \mu \mathrm{M})$ calibrations.

Significant differences in metabolites among the experimental groups were identified using a t-test. Statistical significance was set at $p<0.05$. Principal

A
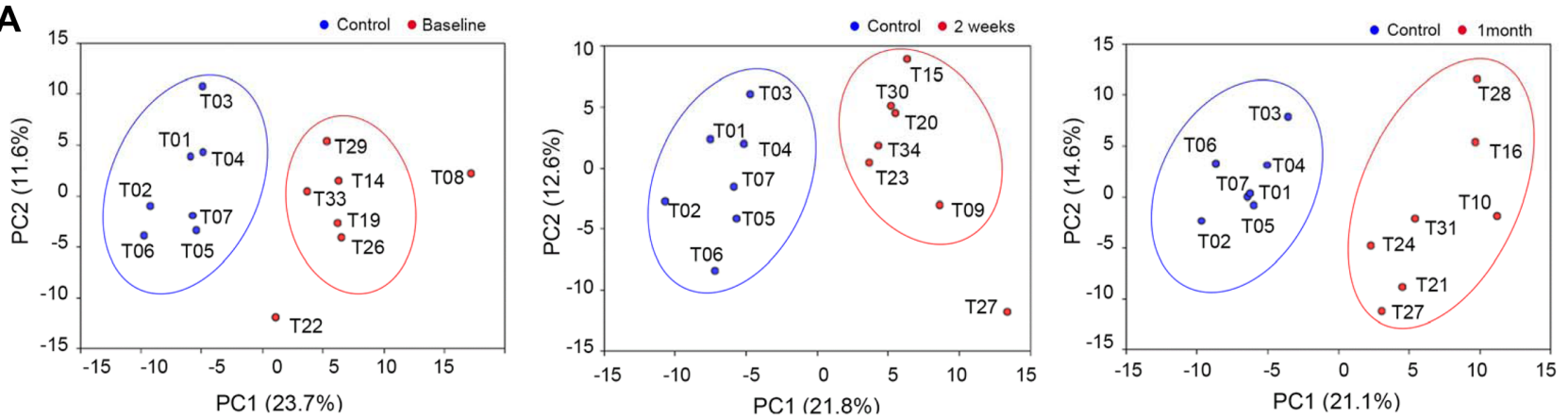

B
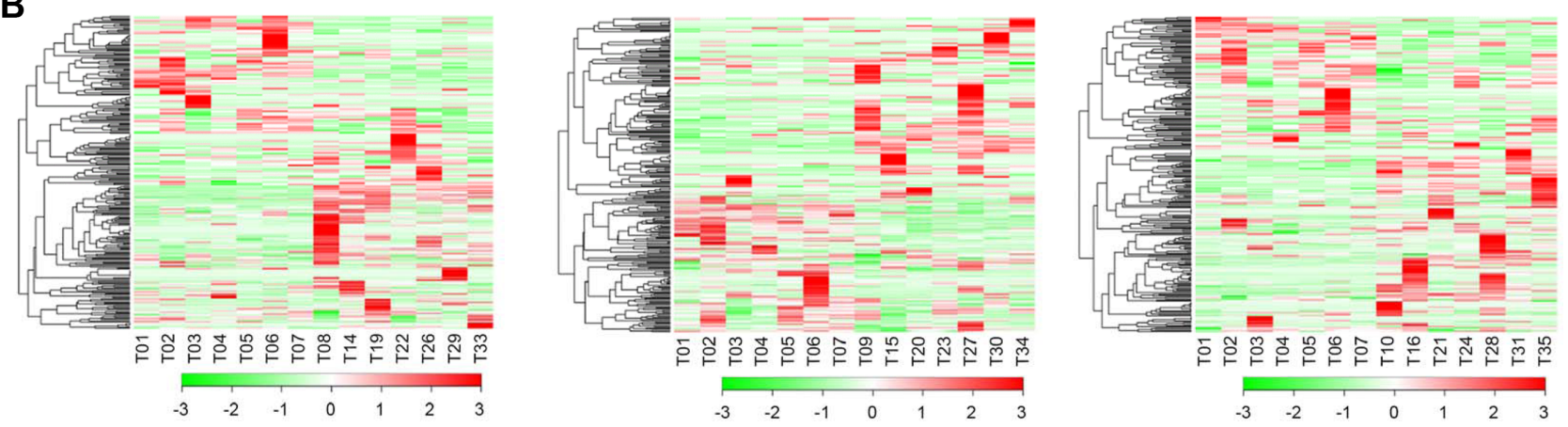

Fig. 1. Principal component analysis and hierarchical cluster analysis. (A) Principal component score plot showing the separation of groups consisting of patients with active tuberculosis (TB) or healthy controls. PC1 and PC2 show the first principal component and second principal component, respectively. The number in parentheses is the contribution rate. Healthy control group: T01, T02, T03, T04, T05, T06, and T07. Baseline group: T08, T14, T19, T22, T26, T29, and T33. The 2 weeks after treatment start group: T09, $\mathrm{T} 15, \mathrm{~T} 20, \mathrm{~T} 23, \mathrm{~T} 27, \mathrm{~T} 30$, and T34. The 1 month after treatment start group: $\mathrm{T} 10, \mathrm{~T} 16, \mathrm{~T} 21, \mathrm{~T} 24, \mathrm{~T} 28, \mathrm{~T} 31$, and $\mathrm{T} 35$. (B) The rows display metabolites and the columns represent samples. 
component analysis (PCA) and hierarchical cluster analysis (HCA) were performed using statistical analysis software developed at HMT.

The TB cohort project collected 21 serum samples from patients with active TB $(n=7)$ and 7 serum samples from healthy people $(n=7)$. Patients were identified as positive for TB based on clinical symptoms classified as a TB infection, a positive MTB smear, and culture. The healthy control group included patients with a negative MTB smear and culture, a negative interferon-gamma release assay, and no TB lesions observed on chest Xray. Patients with active $\mathrm{TB}$ were classified into the following groups according to changes in bacterial load during the first month of treatment: treatment start (baseline), 2 weeks after treatment commencement ( 2 weeks), and 1 month after treatment commencement (1 month).

CE-TOFMS was used to detect mass spectrometry (MS) data in 28 serum samples according to the classification. CE-TOFMS analysis detected and identified 272 peaks based on the HMT standard library. We performed multivariate statistical analysis using the MS data to identify metabolites variation between active TB patients and healthy controls. PCA visualized the relative distance between the two groups. The PCA plot distribution showed a clear variation between the active TB patient group at baseline, 2 weeks after treatment commencement, 1 month after treatment commencement, and healthy controls (Fig. 1A).

Using the profiles of 272 significant differentiating serum metabolites, we drew a heat map to classify the upregulated and downregulated metabolites for TB infection (Fig. 1B). These upregulated and downregulated metabolites may be important for early infection diagnosis and biomarker discovery [15]. The relationship between metabolites in each group is represented by color change. Metabolites with low levels appear in green, while those at high levels are represented in red. The heat map, which standardized the relative area of metabolites, confirmed that a clear cluster was formed between the active TB patient group at baseline, 2 weeks after treatment commencement, 1 month after treatment commencement, and healthy controls. The main mechanisms and substances that appeared between each group were identified by grouping the formed clusters into dendrograms. Through these metabolic changes, the characteristics of each group were confirmed.

A $t$-test was performed to evaluate the statistically significant variation in metabolites in patients across the treatment periods. Compared with healthy controls, there were significant differences in 38 metabolites in the baseline, 44 in the 2 weeks after treatment com-
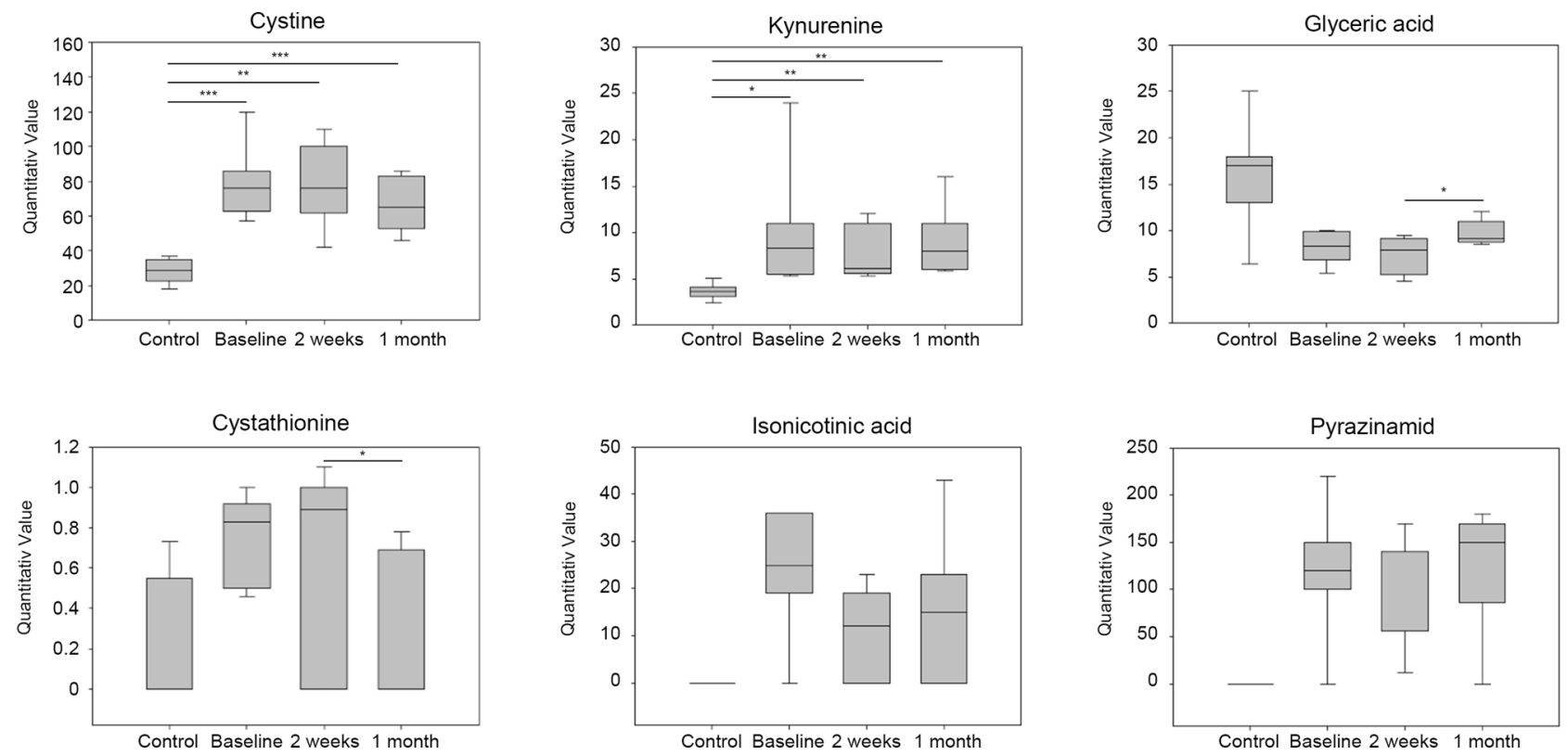

Fig. 2. Dynamic changes in serum metabolite concentrations. Metabolites showing significant differences between the healthy control and tuberculosis treatment groups are shown. ${ }^{*} p<0.05,{ }^{* *} p<0.01$, and ${ }^{* * *} p<0.001$ 
mencement, and 37 in the 1 month after treatment commencement $(p<0.05)$. In addition, according to the patients' treatment, there were significant differences between the baseline and treatment duration groups $(p<0.05)$. Major metabolomic pathways, such as central carbon and amino acid, were identified. Metabolites with significant differences between groups such as cystine, kynurenine, cystathionine, glyceric acid, isonicotinic acid, and pyrazinamide were identified (Fig. 2). Cystine and kynurenine were upregulated in patients with active TB. The ratios of cystine and kynurenine between the groups may be potentially suitable biomarkers to detect active TB infection [16, 17]. Indoleamine 2,3dioxygenase (IDO) has an immunomodulatory function, i.e., it catalyzes tryptophan through the kynurenine pathway. Kynurenine helps suppress the immune response by managing $\mathrm{T}$ cell anergy and proliferation [18]. Additionally, the suitability of sample selection and response to treatment was confirmed by expressing drug-derived metabolites in patients with active TB [19]. Cystathionine was downregulated and glyceric acid was upregulated in the 1 month after treatment commencement group. Using this significant difference in metabolite expression, we identified candidates that can determine the effects of TB treatment [20, 21]. Currently, there have been few studies that have analyzed the metabolism of patients with active TB in South Korea. In this study, through metabolite analysis of patients with active TB in South Korea, serum metabolites that affected TB infection and treatment were identified.

\section{Acknowledgments}

The study was supported by a research grant (2019-NG038-01) of Korea Centers for Disease Control and Prevention.

\section{Conflict of Interest}

The authors have no financial conflicts of interest to declare.

\section{References}

1. Lau SK, Lee KC, Curreem SO, Chow WN, To KK, Hung IF, et al. 2015. Metabolomic profiling of plasma from patients with tuberculosis by use of untargeted mass spectrometry reveals novel biomarkers for diagnosis. J. Clin. Microbiol. 53: 3750-3759.
2. Zumla A, Atun R, Maeurer M, Mwaba P, Ma Z, O'Grady J, et al. 2011. Viewpoint: Scientific dogmas, paradoxes and mysteries of latent Mycobacterium tuberculosis infection. Trop. Med. Int. Health 16: 79-83.

3. Nema V. 2012. Tuberculosis diagnostics: Challenges and opportunities. Lung India 29: 259-266.

4. Gautam US, Foreman TW, Bucsan AN, Veatch AV, Alvarez X, Adekambi T, et al. 2018. In vivo inhibition of tryptophan catabolism reorganizes the tuberculoma and augments immune-mediated control of Mycobacterium tuberculosis. Proc. Natl. Acad. Sci. USA 115: E62-E71.

5. Lange C, Abubakar I, Alffenaar JW, Bothamley G, Caminero JA, Carvalho AC, et al. 2014. Management of patients with multidrugresistant/extensively drug-resistant tuberculosis in Europe: a TBNET consensus statement. Eur. Respir. J. 44: 23-63.

6. Caulfield AJ, Wengenack NL. 2016. Diagnosis of active tuberculosis disease: From microscopy to molecular techniques. J. Clin. Tuberc. Other Mycobact. Dis. 4: 33-43.

7. Moore DF, Curry Jl. 1998. Detection and identification of Mycobacterium tuberculosis directly from sputum sediments by ligase chain reaction. J. Clin. Microbiol. 36: 1028-1031.

8. Odubanjo MO, Dada-Adegbola HO. 2011. The microbiological diagnosis of tuberculosis in a resource - limited setting: is acidfast bacilli microscopy alone sufficient? Ann. Ib. Postgrad. Med. 9: 24-29.

9. Huang H, Shi LY, Wei LL, Han YS, Yi WJ, Pan ZW, et al. 2019. Plasma metabolites xanthine, 4-pyridoxate, and d-glutamic acid as novel potential biomarkers for pulmonary tuberculosis. Clin. Chim. Acta 498: 135-142.

10. Gupta R, Espinal M, Raviglione M. 2004. Tuberculosis as a major global health problem in the 21st century: A WHO perspective. Semin. Respir. Crit. Care Med. 25: 245-253.

11. Harries AD, Kumar AMV. 2018. Challenges and progress with diagnosing pulmonary tuberculosis in low- and middle-income countries. Diagnostics (Basel) 8: 78.

12. Zhang A, Sun H, Yan G, Wang P, Wang X. 2015. Metabolomics for biomarker discovery: Moving to the clinic. Biomed. Res. Int. 2015: 354671.

13. Shah SH, Kraus WE, Newgard CB. 2012. Metabolomic profiling for the identification of novel biomarkers and mechanisms related to common cardiovascular diseases: form and function. Circulation 126: 1110-1120.

14. Wang $X$, Zhang $A$, Han $Y$, Wang $P$, Sun $H$, Song $G$, et al. 2012. Urine metabolomics analysis for biomarker discovery and detection of jaundice syndrome in patients with liver disease. Mol. Cell. Proteomics 11: 370-380.

15. Engle S, Whalen S, Joshi A, Pollard KS. 2017. Unboxing cluster heatmaps. BMC Bioinformatics 18: 63.

16. Adu-Gyamfi CG, Snyman T, Makhathini L, Otwombe K, Darboe F, Penn-Nicholson A, et al. 2020. Diagnostic accuracy of plasma kynurenine/tryptophan ratio, measured by enzyme-linked immunosorbent assay, for pulmonary tuberculosis. Int. J. Infect. Dis. 99: 441-448. 
17. Burns-Huang K, Mundhra S. 2019. Mycobacterium tuberculosis cysteine biosynthesis genes mec+-cys O-cys $M$ confer resistance to clofazimine. Tuberculosis 115: 63-66.

18. Wu H, Gong J, Liu Y. 2018. Indoleamine 2, 3-dioxygenase regulation of immune response (Review). Mol. Med. Rep. 17: 4867-4873.

19. Du Preez I, Loots DT. 2018. Novel insights into the pharmacometabonomics of first-line tuberculosis drugs relating to metabolism, mechanism of action and drug-resistance. Drug Metab. Rev.
50: 466-481.

20. Saini V, Chinta KC, Reddy VP, Glasgow JN, Stein A, Lamprecht DA, et al. 2020. Hydrogen sulfide stimulates Mycobacterium tuberculosis respiration, growth and pathogenesis. Nat. Commun. 11: 557.

21. Mahato S, Saini J, Barabote R. 2020. A putative cystathionine beta-synthase homolog of Mycobacterium smegmatis is involved in de novo cysteine biosynthesis. FASEB J. 34: 1. 